\title{
Detection of the high z GRB 080913 and its implications on progenitors and energy extraction mechanisms ${ }^{\star}$
}

\author{
D. Pérez-Ramírez ${ }^{1,2}$, A. de Ugarte Postigo ${ }^{3}$, J. Gorosabel ${ }^{4}$, M. A. Aloy ${ }^{5}$, G. Jóhannesson ${ }^{6}$, M. A. Guerrero ${ }^{4}$, \\ J. P. Osborne ${ }^{2}$, K. L. Page ${ }^{2}$, R. S. Warwick ${ }^{2}$, I. Horváth ${ }^{7}$, P. Veres ${ }^{7,8}$, M. Jelínek ${ }^{4}$, P. Kubánek ${ }^{4,9}$, S. Guziy ${ }^{4,10}$, \\ M. Bremer ${ }^{11}$, J. M. Winters ${ }^{11}$, A. Riva ${ }^{12}$, and A. J. Castro-Tirado ${ }^{4}$ \\ 1 Departamento de Física, Universidad de Jaén, Campus Las Lagunillas, 23071 Jaén, Spain \\ e-mail: dperez@ujaen.es \\ 2 Department of Physics and Astronomy, The University of Leicester, Leicester, LE1 7RH, UK \\ 3 European Southern Observatory, Casilla 19001, Santiago 19, Chile \\ ${ }^{4}$ Instituto de Astrofísica de Andalucía (IAA-CSIC), PO Box 3.004, 18.080 Granada, Spain \\ 5 Departamento de Astronomía y Astrofísica, Universidad de Valencia, C/ Dr. Moliner s/n, 46100 Burjassot, Valencia, Spain \\ ${ }^{6}$ Department of Physics and SLAC National Accelerator Laboratory, Stanford University, Stanford, CA 94305, USA \\ 7 Dept. of Physics, Bolyai Military University, POB 15, 1581 Budapest, Hungary \\ 8 Dept. of Physics of Complex Systems, Eötvös University, Pázmány P. s. 1/A, 1117 Budapest, Hungary \\ 9 Edif. Institutos de Investigación (GACE-ICMOL), Universidad de Valencia, Campus de Paterna, 46980 Paterna, Valencia, Spain \\ 10 Nikolaev State University, Nikolskaya 24, 54030 Nikolaev, Ukraine \\ 11 Institute de Radioastronomie Milimétrique (IRAM), 300 rue de la Piscine, 38406 Saint Martin d'Héres, France \\ 12 INAF - Osservatorio Astronomico di Brera, Via Emilio Bianchi, 46, Merate, LC, 23807, Italy \\ Received 14 October 2008 / Accepted 14 December 2009
}

\section{ABSTRACT}

\begin{abstract}
Aims. We present multiwavelength observations of one of the most distant gamma-ray bursts detected so far, GRB 080913. Based on these observations, we consider whether it could be classified as a short-duration GRB and discuss the implications for the progenitor nature and energy extraction mechanisms.

Methods. Multiwavelength X-ray, near IR and millimetre observations were made between $20.7 \mathrm{~h}$ and $\sim 16.8$ days after the event. Results. Whereas a very faint afterglow was seen at the 3.5m CAHA telescope in the nIR, the X-ray afterglow was clearly detected in both Swift and XMM-Newton observations. An upper limit is reported in the mm range. We have modeled the data assuming a collimated $\theta_{0} \gtrsim 3^{\circ}$ blast wave with an energy injection at $\sim 0.5$ days carrying $5 \sim 10^{52}$ erg or approximately 12 times the initial energy of the blast wave. We find that GRB 080913 shares many of the gamma-ray diagnostics with the more recent burst GRB 090423 for being classified as short had they ocurred at low redshift. If the progenitor were a compact binary merger, it is likely composed by a $\mathrm{NS}$ and $\mathrm{BH}$. The Blandford-Znajek (BZ) mechanism is the preferred one to extract energy from the central, maximally-rotating BH. Both the magnetic field close to the event horizon $(B)$ and the BH mass $\left(M_{\mathrm{bh}}\right)$ are restricted within a relatively narrow range, such that $\left(B / 3 \times 10^{16} \mathrm{G}\right)\left(M_{\mathrm{bh}} / 7 M_{\odot}\right) \sim 1$. Similar constraints on the central BH hold for collapsar-like progenitor systems if the BZ-mechanism works for the system at hand.
\end{abstract}

Key words. stars: gamma-ray bursts: individual: GRB 080913 - techniques: photometric - Cosmology: observations

\section{Introduction}

Gamma-ray bursts (GRBs) are generally classified into two main groups (Kouveliotou et al. 1993), those with short duration and hard spectra and those with long duration and soft spectra. This simplistic classification scheme could be more complex, as shown by several studies (Zhang et al. 2009; Horváth et al. 2006, 2008).

For short bursts the general idea is that they originate in the near Universe, at redshifts significantly lower $(z \sim 0.5)$ than those of long GRBs (e.g. GRB 060502b at $z=0.287$, Bloom et al. 2007; GRB 051221 a at $z=0.5464$, Soderberg et al. 2006; GRB 050911 at $z=0.1646$, Berger et al. 2007a; GRB 050724 at $z=0.257$, Berger et al. 2005; GRB 050709 at $z=0.160$, Fox et al. 2005; GRB 050509b at $z=0.226$; Gehrels et al. 2005). However, de Ugarte Postigo et al. (2006) observed GRB 060121 $\left(T_{90}=2 \mathrm{~s}\right.$; Arimoto et al. 2006) and provided a most probable

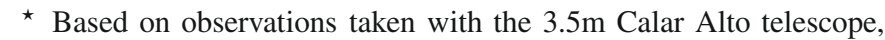
the Plateau de Bure interferometer and the XMM - Newton satellite photometric redshift of $z=4.6$. They suggested that this burst could be the first of a class of short gamma-ray bursts residing at high redshift, which probably belongs to a different progenitor group. Short high-redshift bursts were later studied statistically by Berger et al. (2007b), who found that a significant number of distant short bursts could exist.

GRB 080913 was discovered by Swift on 13 Sep. 2008 (Schady et al. 2008). The burst started at 06:46:54 UT and lasted for $\approx 8 \mathrm{~s}$, placing it, at first sight, in the long-duration class of GRBs (Stamatikos et al. 2008). It was also observed by Konus/WIND and had a fluence of $(5.6 \pm 0.6) \times 10^{-7} \mathrm{erg} \mathrm{cm}^{-2}$ in the $15-150 \mathrm{keV}$ range, making it an average GRB. In the combined BAT-WIND spectrum, the observed prompt energy spectrum could be best-fitted by a power-law with an exponential cutoff model $\mathrm{d} N / \mathrm{d} E \sim E^{\alpha} \times \exp \left(-(2+\alpha) \times E / E_{\text {peak }}\right)$ with $\alpha=-0.89_{-0.46}^{+0.65}$ and $E_{\text {peak }}=131_{-48}^{+225} \mathrm{keV}$ (Pal' shin et al. 2008).

The prompt dissemination (21 s) of the GRB position by Swift enabled instant responses of robotic telescopes, such as the REM robotic telescope (D'Avanzo et al. 2008). Rapid 
observations obtained by GROND at the $2.2 \mathrm{~m}$ telescope in La Silla allowed the identification of a near-infrared (nIR) counterpart (Rossi et al. 2008) 3 min after the burst trigger. Shortly after Swift slewed and started data acquisition, a fading X-ray source was detected by the Swift/XRT, which was identified as the GRB 080913 afterglow (Beardmore et al. 2008). This triggered a multiwavelength campaign at different observatories aimed at studying the afterglow. A photometric redshift in the range 6.1-6.7 was derived (Greiner et al. 2008), and a spectroscopic $z=6.7$ was later confirmed by a VLT spectrum (Fynbo et al. 2008, Greiner et al. 2009). This implied rest frame values of $T_{90} \sim 1 \mathrm{~s}$ and $E_{\text {peak, rest }} \sim 1000 \mathrm{keV}$ consistent with a short-duration GRB (Pal'shin et al. 2008). This is also supported by the negligible spectral time lags found in the BAT energy range (Xu 2008). For a standard cosmology model with $\mathrm{H}_{0}=71 \mathrm{~km} \mathrm{~s}^{-1} \mathrm{Mpc}^{-1}, \Omega_{\mathrm{M}}=0.27, \Omega_{\Lambda}=0.73$, the isotropic energy release is $E_{\text {iso }} \sim 7 \times 10^{52} \mathrm{erg}(1 \mathrm{keV}-10 \mathrm{MeV}$, rest frame), with a look-back time of $13.67 \mathrm{Gyr}$.

A more recently detected burst, GRB 090423 (Tanvir et al. 2009, Salvaterra et al. 2009), is a further extreme redshift $(z=8.2)$ and potentially short GRB which exhibits similar properties, such as burst duration $\left(T_{90} \sim 10.3 \pm 1.1 \mathrm{~s}\right.$, and a rest-frame duration of $\sim 1 \mathrm{~s}$ ), spectral lag times negligibly small, consistent with zero, and an intrisincally hard spectrum as GRB 080913.

This burst, together with GRB 080913, points to the fact that the current dichotomy is not always consistent. That is, standard indicators of the physical nature of GRBs, such as duration and hardness, may no longer be the only diagnostic used in physically classifying high-redshift GRBs. They reveal a need for a revision of the traditional observational criteria. Zhang et al. (2009) tackled this question proposing new operational procedures in the determination of the physical category of GRBs. According to this work, GRB 080913 and GRB 090423 are considered to belong to the type II category (i.e. massive-star core collapse origin). However, these two high- $z$ bursts may also be compatible with a "specific type I scenario" driven by the Blanford-Znajek mechanism in a BH-NS merger.

Here we report multiwavelength observations carried out, from the millimetre to the X-ray band, in order to study the afterglow of GRB 080913. We also discuss the implications of these observations for the nature of short-duration GRBs progenitors. Finally, we include some results extracted from the literature about the implications for the nature of long-duration GRBs progenitors

\section{Observations and data reduction}

\subsection{Near-IR observations}

Target of opportunity (ToO) observations in the nIR were triggered starting $20.7 \mathrm{~h}$ after the event with the $3.5 \mathrm{~m}$ telescope (+OMEGA 2000; Bailer-Jones et al. 2000) at the German-Spanish Calar Alto Observatory (CAHA). A $5500 \mathrm{~s} \mathrm{im-}$ age was acquired in the $J$-band filter (see Fig. 1), with a $1.35^{\prime \prime}$ average seeing. We followed the standard data reduction procedures such as dark and sky frame subtraction, plus bad-pixel mask and master flat-field correction. The photometry for our final image was performed by means of the PHOT routine under IRAF $^{1}$. A range of apertures were checked, and the one yielding the minimum photometric error was selected. The candidate initially reported by Rossi et al. (2008) was barely detected $(2.3 \sigma$

\footnotetext{
${ }^{1}$ IRAF is distributed by the NOAO, which are operated by USRA, under cooperative agreement with the US NSF.
}

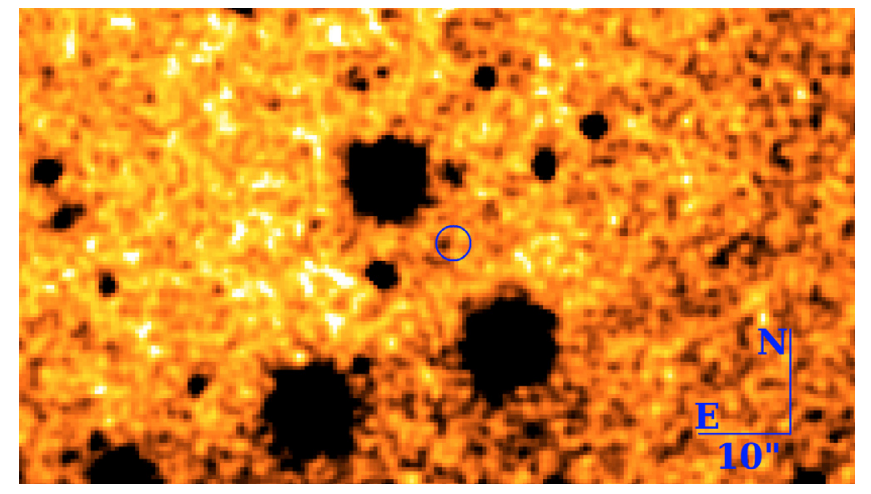

Fig. 1. The $J$-band image of the GRB 080913 field taken at the $3.5 \mathrm{~m}$ CAHA telescope on 14 Sep. 2008 (20.7 h after the burst onset). The error circle marks the XRT position of the counterpart with a radius of 1.9" (Beardmore et al. 2008).

level) in the $J$-band image (Fig. 1), with an estimated $J$-band Vega magnitude of $22.4 \pm 0.5$, including the calibration zero point error $(0.15 \mathrm{mag})$ given by the 2 MASS Catalogue. In order to compare our detection with the lightcurve for this GRB as presented by Greiner et al. (2009), we have evaluated the AB-toVega system magnitude offset coefficient for the OMEGA 2000 instrument in the $J$-band to be 0.97 . The converted J-band magnitude in the $\mathrm{AB}$ system for our detection is $23.4 \pm 0.5$, which at the time of the observation, agrees with the GROND data (Greiner et al. 2009). Our detection occurred close to the peak of the re-brightening phase (Fig. 4).

\subsection{Millimetre observations}

Additional $\mathrm{mm}$ observations were obtained at the Plateau de Bure Interferometer (PdBI) as part of our ToO programme. The PdBI observed the source on different occasions in the period of time of three days in compact configuration. We used the carbon star MWC349 as primary flux calibrator (assuming $\left.F(v)=1.1 \cdot(v / 86.2 \mathrm{GHz})^{0.6}\right)$ with the amplitude and phase calibrations relative to the quasar 0454-234. The data reduction was done with the CLIC and MAPPING software distributed by the Grenoble GILDAS group. We analysed the data with positionfixed $(\operatorname{RA}(2000)=04: 22: 54.66, \operatorname{Dec}(2000)=-25: 07: 46.2)$ fits in the UV plane, which only yielded upper limits but allows to constrain the mm-lightcurve. The $3 \sigma$-limits are $0.72 \mathrm{mJy}$ (99 GHz, Sep 16.1 UT), 1.44 Jy (84 GHz, Sep $21.2 \mathrm{UT}$ ) and 0.90 mJy (106 GHz, Sep 30.1 UT).

\subsection{X-ray observations}

We made use of public Swift/XRT data obtained starting 94 s after the BAT trigger time $\left(T_{0}\right)$. The data were acquired in the XRT Photon Counting (PC) mode.

The lightcurve in the $0.3-10 \mathrm{keV}$ energy range (Evans et al. 2007) exhibits a number of flares in the initial orbit, with the peak of the largest flare observed at $T_{0}+1800 \mathrm{~s}$ (in the observer frame). The decay in the time interval $T_{0}+400 \mathrm{~s}$ to $T_{0}+1100 \mathrm{~s}$ can be fitted by a power-law with an estimated decay index $\alpha_{X}=1.2_{-0.1}^{+0.2}$ (where $f_{\mathrm{X}} \propto t^{-\alpha_{\mathrm{X}}}$ ). The spectrum corresponding to the period of flare activity in the initial orbit, can be fitted by an absorbed power-law with a photon index of $\Gamma \sim 1.7_{-0.4}^{+0.5}$, assuming that the Galactic column density value in the direction of the burst is $3.2 \times 10^{20} \mathrm{~cm}^{-2}$ (Kalberla et al. 2005).

A ToO XMM-Newton observation (Obs. ID. 0560191701) started on Sep 17.61 UT, i.e. 4.3 days after the Swift/BAT trigger. 


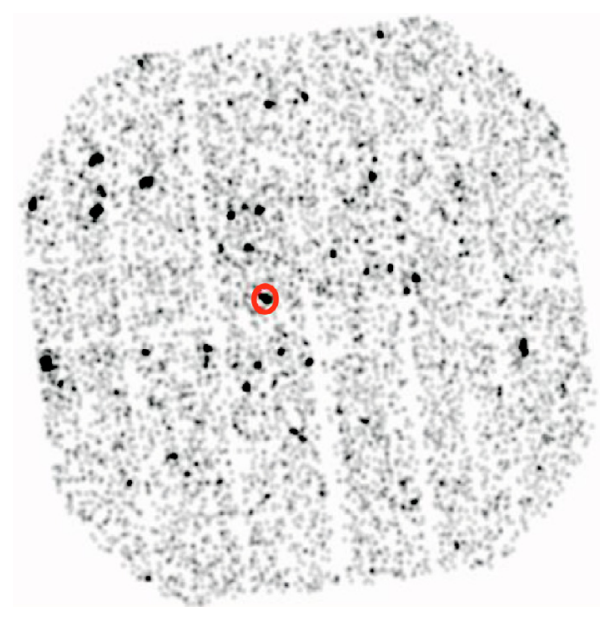

Fig. 2. The EPIC-pn image of the high redshift GRB 080913 obtained with XMM-Newton $\sim 4.5$ days after the burst onset. The image has been adaptively smoothed to emphasize the detection of the GRB. The position derived by Swift for this burst is marked by the circle.

The European Photon Imaging Camera (EPIC) CCD cameras were operated in the Full Frame Mode. The total exposure time for the EPIC-pn camera was $14.0 \mathrm{ks}$. The Thin 1 optical blocking filter was used for the EPIC-pn camera, whereas the medium filter was used for both EPIC-MOS cameras. The XMM-Newton Observation Data Files (ODF) were processed using XMMNewton Science Analysis Software (SAS version 7.1.0) and the calibration files from the Calibration Access Layer as on $14 \mathrm{Dec}$. 2007. After excising periods of high-background, the net exposure times of the EPIC-pn, MOS1, and MOS2 observations are reduced to $3.6 \mathrm{ks}, 10.8 \mathrm{ks}$, and $7.9 \mathrm{ks}$, respectively.

The XMM-Newton EPIC observations of GRB 080913 detected X-ray emission from its afterglow (see Fig. 2) at EPIC-pn, MOS1, and MOS2 count rates of $0.0109 \pm 0.0021 \mathrm{cnts} \mathrm{s}^{-1}$, $0.0032 \pm 0.0008 \mathrm{cnts} \mathrm{s}^{-1}$, and $0.0019 \pm 0.0008 \mathrm{cnts} \mathrm{s}^{-1}$, respectively. The net count number, $\sim 90$ counts, is not sufficient to carry out a spectral fit. Adopting an absorbed power-law model of spectral index $\Gamma=1.7$ and column density $N_{\mathrm{H}}=3.2 \times$ $10^{20} \mathrm{~cm}^{-2}$, the EPIC spectral shapes and count rates imply an absorbed X-ray flux of $3.8 \times 10^{-14} \mathrm{ergs} \mathrm{cm}^{-2} \mathrm{~s}^{-1}$ and an unabsorbed X-ray flux of $4.1 \times 10^{-14} \mathrm{ergs} \mathrm{cm}^{-2} \mathrm{~s}^{-1}$ in the energy band $0.3-10 \mathrm{keV}$. The X-ray luminosity in this same band at restframe is $5.7 \times 10^{45} \mathrm{erg} \mathrm{s}^{-1}$ (assuming in XSPEC $H_{0}=71 \mathrm{~km} \mathrm{~s}^{-1} \mathrm{Mpc}^{-1}$ and $\left.\Omega_{\Lambda}=0.73\right)$.

\section{Results and discussion}

Following the discovery of the X-ray afterglow with Swift, we detected a faint $\mathrm{nIR}$ afterglow (consistent with the position given by Rossi et al. 2008) and the X-ray afterglow 4.5 days after with $X M M-N e w t o n$. We discuss in this section the classification and likely progenitor of this burst.

\subsection{Spectral Flux Distribution of GRB 080913}

Using the model and methods described by Jóhannesson et al. (2006) we fitted the multiband observations of the afterglow to a fireball model with energy injections.

One injection is needed in order to account for the bump seen at $\sim 0.5$ days in the light curves. Since no jet-break is seen in the light curves up to around 10 days, we can only put a lower limit to the collimation angle of the jet, $\theta_{0} \gtrsim 3^{\circ}$. This is delayed from the definition of Sari et al. (1999) due to the energy injection,

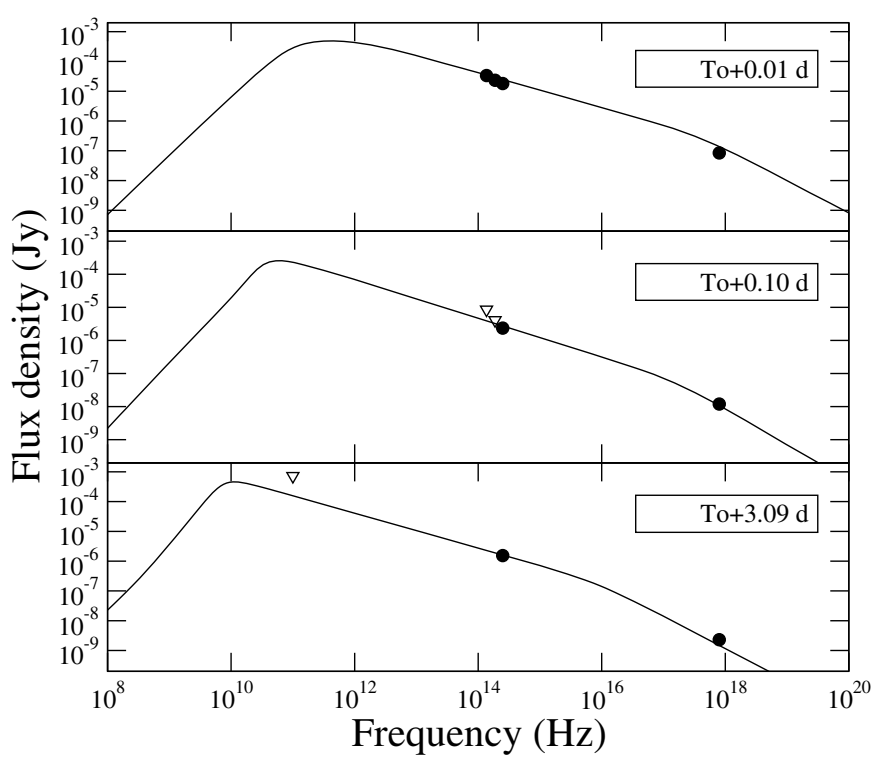

Fig. 3. Spectral Flux Distribution of the afterglow from radio to X-rays $0.01,0.10$, and 3.09 days after the burst in the observer frame. Filled circles are based on the nIR data from Greiner et al. (2009) and the X-ray data from Swift/XRT. Triangles represent upper limit: in the $\mathrm{T}_{0}+$ 0.01 days plot they correspond to nIR observation upper limits whereas in the $T_{0}+3.09$ days plot it is the $3-\sigma$ upper limit obtained at PdB (this paper).

the sideways expansion of the jet and the detailed calculation of the equal arrival time surface. Our preferred scenario (giving the best fit) for a collimation of $3^{\circ}$ is an initial energy release of $E_{0}=4 \times 10^{51}$ erg into a uniform medium with density $n_{0}=2 \mathrm{~cm}^{-3}$. This fit results in a $\chi^{2} /$ d.o.f. $=170 / 50$ where the high value is mainly caused by the scatter in the X-ray light curve the model is unable to reproduce. In order to explain the bump at 0.5 days, an energy injection carrying approximately $12 E_{0}\left(5 \times 10^{52} \mathrm{erg}\right)$ is needed. Due to the lack of a turnover in the light curves at early time, we require the initial Lorentz factor of the blast wave to be $\Gamma_{0} \gtrsim 500$. The SFD is best fit with an electron index $p=2.17$ and micro- physical parameters $\epsilon_{\mathrm{i}}=6 \times 10^{-4}$ and $\epsilon_{\mathrm{B}}=3 \times 10^{-5}$. Note that the parametrization of the electron population has changed from Johannesson et al. (2006) and we now follow the tradition of Panaitescu \& Kumar (2001). The minimum Lorentz factor of the electron distribution is now defined as $\gamma_{\text {min }}=\epsilon_{\mathrm{i}} m_{\mathrm{p}} / m_{\mathrm{e}}(\Gamma-1)$. Figure 3 shows the radio to X-ray SFD predicted by our model for 3 epochs together with observation data corrected for intrinsic extinction. The Galaxy extinction is negligible.

Please note the the above values for the best fit parameters are in many cases highly dependent on the value chosen for $\theta_{0}$. The energy required for the blast wave goes as $\theta_{0}^{2}$ and lower values of $\theta_{0}$ put an upper limit on $n_{0}$ from the jet break requirements. Additionally, there is a strong correlation between the value of $n_{0}$ and $\epsilon_{\mathrm{B}}$ because we do not have the required data to constrain the SFD at lower frequencies. Other parameters are less sensitive to the value of $\theta_{0}$, especially $p$ that is well constrained from the nIR SFD.

\subsection{Comparison with high-redshift long GRB 050904 and short GRB 060121}

Given their highest redshifts, one could qualitatively compare the properties of GRB $080913(z=6.7)$, GRB $060121(z=4.6)$ and GRB $050904(z=6.295)$. The reason for choosing the latest 


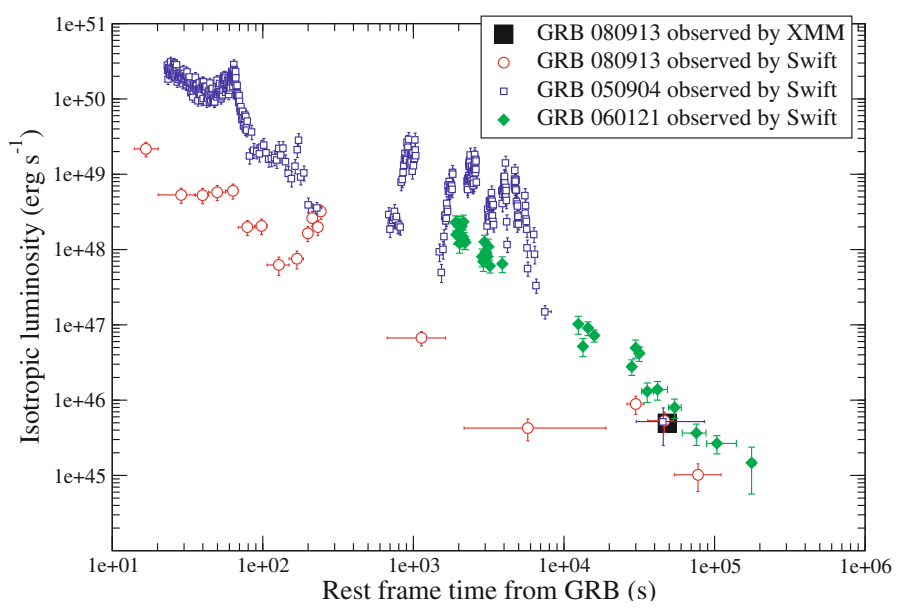

Fig. 4. The K-corrected (rest frame) $0.3-10 \mathrm{keV}$ isotropic luminosity evolution of GRB 080913 (red) in comparison to the long-duration GRB 050904 (blue, $z=6.3$ ) and the short-duration GRB 060121 (green, $z=4.6$ ). The solid square at $t-t_{\mathrm{GRB}}=4.9 \times 10^{4} \mathrm{~s}$ represents the XMM-Newton observations carried out for GRB 080913. As seen GRB 080913 shows lower isotropic luminosities than the short GRB 060121.

cases is that GRB 060121 is a short duration GRB $\left(T_{90} \sim 2 \mathrm{~s}\right)$, very likely at high-redshift $(z=4.6$, de Ugarte Postigo et al. 2006), whereas GRB 050904 is a long GRB $\left(T_{90} \sim 31 \mathrm{~s}\right)$ at a comparable redshift $(z=6.295$; Haislip et al. 2006; Kawai et al. 2006). We have constructed the restframe isotropic $0.3-10 \mathrm{keV}$ luminosity lightcurves of the three GRBs, assuming a power law spectrum with a photon index $\Gamma$. The time evolution of $\Gamma$ was found by using linear interpolation between the $\Gamma$ values determined from Swift /XRT spectra on a logarithmic time scale. This was done to get a smooth K-correction with time. As seen in Fig. 4 the $0.3-10 \mathrm{keV}$ decay of GRB 080913 is similar in character to those exhibited by GRB 060121 and GRB 050904. However, its isotropic luminosity at early stages is lower by a factor $\sim 30$. Therefore, based on the afterglow lightcurve it is not possible to rule out any of the possible origins for the progenitor.

\subsection{Implications for a short duration progenitor origin}

Because of the intrinsically short duration, hard spectrum and the effectively zero spectral lags of GRB 080913, we consider in the following the constraints on the typical progenitor systems invoked for standard short-hard GRBs. We note that a late energy injection, used to fit the afterglow light curve (Sect. 3.1), does not necessarily argues against a short GRB progenitor or, equivalently, imply a long lived central engine, if the ejected material has a distribution in initial Lorentz factors. Under the assumption that the initial material powering the afterglow was ejected with a Lorentz factor of $\sim 500$, matter ejected simultaneously with a Lorentz factor of $\sim 40$ would refresh the afterglow at around 0.5 days. The rather large energy injection of 10 times the initial energy release requires, however, a significant increase in the efficiency of the central engine that poses constraints on either short or long lived central engines.

At a redshift $z=6.7$, the burst ocurred when the Universe was only $\sim 0.8$ Gyr old. According to Yoshida et al. (2008), the first stars formed $\sim 0.2$ Gyr after the Big Bang. Thus, if GRB 080913 resulted from a merger of compact objects, the whole evolution of the progenitor stars $\left(\tau_{\text {evol }}\right)$ and the merger time $\left(\tau_{\text {mer }}\right)$ happened within $\tau=\tau_{\text {evol }}+\tau_{\text {mer }} \lesssim 0.6$ Gyr. Such a small value of $\tau$ sets a lower limit to the mass of the progenitor stars. Thus, neither of the two stars forming the binary system could be less massive than $\sim 4 M_{\odot}$. This only restricts the type of progenitor system if the burst were a result of the merger of a white dwarf (WD) and a BH. ${ }^{2}$

As the lower accretion timescales $(\sim 10 \mathrm{~s})$ occur for the higher-mass WD (Popham et al. 1999), the WD should have a mass close to the Chandrasekar mass $\left(\sim 1.4 M_{\odot}\right)$. For $\mathrm{NS}+\mathrm{NS} / \mathrm{BH}$ binaries, the evolution of the progenitor stars is only a small fraction of $\tau\left(\tau_{\text {evol }} \sim 10-20\right.$ Myr; Belczynski et al. 2006). Thus, $\tau$ roughly equals $\tau_{\text {mer }}$ in this case.

Since $\tau_{\text {mer }} \sim 0.6 \mathrm{Gyr}$, if the GRB originated from a double NS (DNS) merger, it could have proceeded through a classical merger channel, for which $\tau_{\text {mer }} \sim 0.1-15$ Gyr (Belczynski et al. 2006). Considering the large $E_{\text {iso }}$ of GRB 080913, it is more likely that it was hosted in a NS+BH merger than in a DNS. The latter type of mergers have typically $\tau_{\mathrm{mer}}^{\mathrm{NS}+\mathrm{BH}} \sim 1 \mathrm{Gyr}$, but with a non-negligible merger probability for times $0.1 \mathrm{Gyr} \lesssim \tau_{\text {mer }}^{\mathrm{NS}+\mathrm{BH}} \lesssim$ 1 Gyr.

According to Oechslin \& Janka (2006) the total equivalent isotropic energy released in gamma-rays $\left(E_{\text {iso }}\right)$ from a progenitor consisting of a $\mathrm{BH}$-accretion torus system, which boosts a neutrino driven ultrarleativistic jet is

$E_{\text {iso }}=f_{1} f_{2} f_{3} f_{4} f_{\Omega}^{-1} M_{\text {acc }} c^{2}$,

where $M_{\text {acc }}$ and $c$ are the accreted mass and the light speed, respectively, $f_{1}, f_{2}, f_{3}$, and $f_{4}$ are different efficiency factors (see below), and $f_{\Omega} \simeq \theta_{0}^{2} / 2$ is the jet collimation factor. For GRB 080913, both $f_{\Omega}$ and $f_{4}$, the fraction of the energy of ultrarelativistic jet matter, which can be emitted in gamma-rays in course of dissipative processes that occur in shocks, can be fixed from the light curve fit shown in Sect. 3.1. A jet collimation factor $f_{\Omega}=1.37 \times 10^{-3}$ results taking as jet half-opening angle $\theta_{0}=3^{\circ}$ (Sect. 3.1). This value is smaller than, but consistent with $f_{\Omega} \sim 0.015-0.034$ obtained in numerical models (Aloy et al. 2005), since $\theta_{0}$ is only a lower bound in our case. The factor $f_{4}$ is set by the ratio $f_{4}=f_{\Omega} E_{\gamma} / E_{\mathrm{AG}}=0.024$, where $E_{\mathrm{AG}}$ is taken here equal to the kinetic energy used to model initial afterglow in Sect. 3.1, i.e., $E_{\mathrm{AG}}=E_{0}$. Such figure is also consistent with estimates of the internal shock model, $f_{4} \lesssim 0.3$ (e.g., Mimica \& Aloy 2010, and references therein). For the remaining factors in Eq. (1), we find that the observed energy in the prompt GRB phase needs the concurrence of a large accretion disk mass $\left(M_{\text {acc }} \gtrsim 0.55 M_{\odot}\right.$; Oechslin et al. (2007) obtain $\left.M_{\text {acc }} \lesssim 0.3 M_{\odot}\right)$ and several large efficiency factors (probably so large that they rule out a neutrino mediated energy extraction from the central engine). A large conversion efficiency of the accreted mass into neutrino emission $f_{1} \gtrsim 0.1$ (to be compared with a typical value $f_{1} \gtrsim 0.05$; e.g. Setiawan et al. 2006; Lee et al. 2005), a conversion efficiency of neutrinos and antineutrinos by annihilation to $\mathrm{e}^{ \pm}$pairs $f_{2} \gtrsim 0.06$ (for reference, $f_{2} \sim 0.001, \ldots, 0.04$ is estimated by Ruffert \& Janka (1999), Setiawan et al. (2006), or Birkl et al. (2007), and a large fraction of the $\mathrm{e}^{ \pm}$-photon fireball energy which drives the ultra-relativistic outflow $f_{3} \gtrsim 0.4$ (larger than $f_{3} \sim 0.1$ extracted from simulations; Aloy 2005). The combination of a large $f_{2}$ and a large disk mass is supported by the steady models of Birkl et al. (2007) with values of the dimensionless angular momentum of the central BH $a \sim 0.4-0.5$.

We note that if $E_{\mathrm{AG}}$ is taken to be the sum of the contributions due to the initial kinetic energy $\left(E_{0}\right)$ and of the late energy injection (12E $E_{0}$; Sect. 3.1), then $f_{4} \sim 1.3 \times 10^{-3}$, implying that all the remaining free efficiency factors $\left(f_{1}, f_{2}\right.$ and $\left.f_{3}\right)$ have to be, at least, a factor of 5 larger than the largest estimates of them,

\footnotetext{
2 WD+NS mergers possess much longer timescales and are more likely progenitors of long GRBs (e.g., King et al. 2007)
} 
which suggests that a neutrino driven outflow cannot account for the observed phenomenolgy.

We point out that the previous analysis is only sensitive to the value of $\theta_{0}$ indirectly, if we seek to accommodate with our model the values of $E_{\mathrm{AG}}$. This is because $E_{\mathrm{AG}} \simeq f_{1} f_{2} f_{3} M_{\mathrm{acc}} c^{2}$, but the fitted values of $E_{\mathrm{AG}}$ depend on $\theta_{0}^{2}$ (Sect. 3.1). Berger (2007) makes the hypothesis that outflows of short-duration GRBs with the highest energies are strongly collimated. If the outflow is neutrino-driven, we point out that such hypothesis seems consistent with the fact that the largest $E_{\text {iso }}$ are linked to BH-torus systems in which either the torus mass is large or $f_{1}$ is large. A large torus mass may arise in mergers between compact objects of different masses (Oechslin \& Janka 2006; Shibata \& Taniguchi 2008). The conversion efficiency of the accreted mass into neutrino emission tends to increase with increasing values of the torus viscosity (Setiawan et al. 2006). Remarkably, a large viscosity yields more vertically extended (inflated) accretion tori, which may help to collimate the ultra-relativistic outflow in narrow channels.

Given the large efficiencies needed to account for the large $E_{\text {iso }}$ of GRB 080913 if the outflow were neutrino driven, it seems more natural in this subclass of extremely energetic short GRBs to invoke an energy extraction mechanism directly linked to the BH spin, e.g., the Blandford-Znajek (BZ) process (Blandford $\&$ Znajek 1977). In this case, an estimate of the total power produced by the central engine is (Lee et al. 2000), $P_{\mathrm{BZ}}=$ $1.7 \times 10^{50} a^{2}\left(B / 10^{15} \mathrm{G}\right)^{2}\left(M_{\mathrm{bh}} / M_{\odot}\right)^{2} f(h)$, where $f(h)=[(1+$ $\left.\left.h^{2}\right) / h^{2}\right][(h+1 / h) \arctan (h)-1], H=a /\left(1+\sqrt{\left(1-a^{2}\right)}\right.$, and $B$ is the magnetic field strength at the event horizon of the $\mathrm{BH}$ with mass $M_{\mathrm{bh}}$. We obtain the isotropic equivalent energy released in $\gamma$-rays due to this process during the intrinsic event duration $\left(T_{90} \simeq 1 \mathrm{~s}\right)$ as $E_{\text {iso }}=P_{\mathrm{BZ}} * T_{90} * f_{3} * f_{4}$. Note that in the previous estimate, we use the same efficiency factors $f_{3} \sim 0.4$ and $f_{4}=0.024$ as in the previous paragraphs to account for the facts that (1) only a fraction of the released energy will be used to drive a ultrarelativistic outflow, and that (2) the radiated energy in $\gamma$-rays is much smaller than the kinetic energy of the outflow $E_{\mathrm{AG}}$. Hence, the observed energy can be reached if either the value of the dimensionless angular momentum of the central $\mathrm{BH}$ is $a \sim 1$, the magnetic field surrounding the $\mathrm{BH}$ is $B \gtrsim 10^{16} \mathrm{G}$ or the $\mathrm{BH}$ has a mass $M_{\mathrm{bh}} \gtrsim 20 M_{\odot}$. We note that to form a $20 M_{\odot} \mathrm{BH}$ in a low-metallicity star (which shall be the case at the redshift of GRB 080913), the initial mass of such star shall be $\gtrsim 50 M_{\odot}$ (Woosley et al. 2002).

Since $P_{\mathrm{BZ}}$ depends quadratically on both $B$ and $M_{\mathrm{bh}}$, we may estimate which is the range of variation of these two parameters such that the resulting $E_{\text {iso }}$ complies with the energetics observed for GRB 080913. Lower values of the BH mass, $M_{\mathrm{bh}} \sim 3 M_{\odot}$, closer to the typically considered ones in mergers of compact objects, require extremely large values of the magnetic field strength $\left(B \gtrsim 7 \times 10^{16} \mathrm{G}\right)$. Such magnetic fields would probably brake excesively the rotation of the stellar progenitor core, likely inhibiting the formation of a maximally rotating Kerr $\mathrm{BH}$ (i.e., reducing the value of $a$ sensitively below 1). Hence, in account of the large estimated mass of the $\mathrm{BH}$, if a merger of compact objects were the progenitor system of this GRB, a $\mathrm{NS}+\mathrm{BH}$ merger is favoured, since the typical mass of the $\mathrm{BH}$ resulting from a DNS merger is $\lesssim 3 M_{\odot}$. On the other hand, if we consider values of the magnetic field strengh smaller than the reference value of $10^{16} \mathrm{G}$, we find that even a factor of three smaller field yields a $\mathrm{BH}$ mass $M_{\mathrm{bh}} \sim 60 M_{\odot}$. To form such a massive $\mathrm{BH}$ the progenitor star should have a mass $\sim 140 \mathrm{M}_{\odot}$, i.e., in the limit of being stars which are disrupted at the end of their lives by the pair instability without leaving any remnant BH (Woosley et al. 2002). Thus, both the magnetic field strength and the $\mathrm{BH}$ mass are restricted within a relatively narrow range, $3 \times 10^{15} \mathrm{G} \lesssim B \lesssim 3 \times 10^{16} \mathrm{G}$, and $7 M_{\odot} \lesssim M_{\text {bh }} \lesssim 60 M_{\odot}$, respectively. Within the former range of values for the triad of parameters $a, B$ and $M_{\mathrm{hb}}$, the most likely ones are those favouring the largest possible value of $a$. Because of the fact that strong magnetic fields tend to slow down the rotation of the stellar core, and because of the difficulty to build up magnetic fields in excess of $\sim 10^{15} \mathrm{G}$ by the collapse of stellar cores (e.g., Obergaulinger et al. 2006a,b), even considering the action of the magnetorotational instability (Akiyama et al. 2003; Obergaulinger et al. 2009), values of $a \sim 1$ fit better with the lowest values of $B$ in the aforementioned range. Thereby, to reach the appropriate BZ power to fuel GRB 080913, we favour BH masses in the upper end of the interval stated above.

As noted above, if the total energy of the afterglow is $E_{\mathrm{AG}} \simeq$ $13 E_{0}$, the reduced value of $f_{4}$, yields even more stringent constraints on the central engine, since $P_{\mathrm{BZ}}$ has to be 13 times larger, which needs of $a \sim 1, B \gtrsim 1.6 \times 10^{16} \mathrm{G}$ and $M_{\mathrm{bh}} \gtrsim 50 M_{\odot}$. However, we have to be cautious with the inferences based on the values of the total kinetic energy in the afterglow, since differently from $E_{\text {iso }}$ (directly measured), $E_{\mathrm{AG}}$ results from a model fit of the afterglow light curve, which sensitively depends on the value of $\theta_{0}$ (Sect. 3.1).

Finally, the large redshift of GRB080913 fits in theoretical models where the rate of NS+NS/BH mergers follows either the star formation rate or the star formation rate with delays smaller than 1 Gyr (Janka et al. 2006).

\subsection{Implications for a long duration progenitor origin}

Recent studies (Zhang et al. 2009; Belczynski et al. 2009) have suggested that GRB 080913 has a long-duration progenitor origin.

Zhang et al. (2009) present a new scheme for classifying bursts based on criteria more closely related to the progenitor type to differentiate physical origins, such as $\mathrm{SN}$ association, host galaxy properties and the offset of the GRB location in the host galaxy. They classify bursts into two main categories: type I (with a compact star-merger origin) and type II (with a massivestar core collapse origin). Under such scheme, GRB 080913 and GRB 090423 are identified as type II candidates based on (i) the geometrically-corrected gamma-ray $\left(E_{\gamma}\right)$ and kinetic $\left(E_{\mathrm{K}}\right)$ energies (with large values); (ii) intrinsic afterglow luminosities (moderately bright); (iii) the high density of the circumburst medium and, (iv) the marginal compliance of the $E_{\mathrm{p}}-E_{\gamma \text {, iso }}$ relation. However, because these bursts are intrinsically short, but still considered more likely long, they suggested a possible "specific type I scenario" driven by the Blandford-Znajek mechanism of a BH-NS merger.

The analysis made in the previous section, regarding the properties of the central $\mathrm{BH}$ in order to be able to deliver the sought $E_{\text {iso }}$ of GRB 080913, is formally independent of the fact that the progenitor system is a single massive star or a member of a binary. Thus, we cannot exclude the possibility that the progenitor system is a massive low-metallicity star that forms a collapsar-like engine (or a type II GRB according to the classification of Zhang et al. 2009) whose energy is extracted by means of a BZ-mechanim. In such a case, the estimated BH mass and magnetic field are the same as in Sect. 3.3.

Belczynski et al. (2009) point out that based on the currently used gamma-ray diagnostics $\left(T_{90}, E_{\text {peak }}\right.$, hardness ratio) these 
bursts would be considered as short had they occured at low redshift. They argue that based on the average Swift detection rates (accounting for selection effects), estimated for long GRBs to exceed 10 times the rates for short GRBs, these bursts might belong to the long class. At the redshifts of these GRBs, the calculated rates are $1 \mathrm{yr}^{-1}$ and $0.1 \mathrm{yr}^{-1}$ per unit redshift, for long and short GRBs, respectively.

\section{Conclusions}

We report multiwavelength observations of the high $z$, potentially short-duration gamma-ray burst GRB 080913 acquired between $20.7 \mathrm{~h}$ and 16.8 days after its detection by Swift. Although nIR and X-ray afterglows were found, no $\mathrm{mm}$ afterglow was detected. The X-ray spectrum is consistent with negligible intrinsic absorption. We have modeled the data with a collimated $\left(\theta_{0} \gtrsim\right.$ $3 \mathrm{deg}$ ) blast wave with an energy injection at 0.5 days, requiring a total energy release of more than $5 \times 10^{52} \mathrm{erg}$.

At a redshift $z=6.7$, the burst ocurred when the Universe was only $\sim 0.8$ Gyr old. If GRB 080913 resulted from a merger of compact objects, the whole evolution of the progenitor stars and the merger time happened within $\lesssim 0.6 \mathrm{Gyr}$, following the star formation rate with delays of $\lesssim 1$ Gyr (Janka et al. 2006). This implies that neither of the two stars could be less massive than $\sim 4 M_{\odot}$. If the system of compact binaries were formed by a WD and a BH, the short duration of the burst restricts the mass of the WD to be close to the Chandrasekar mass. If the progenitor system of this GRB is a merger of compact objects, a NS+BH system is favored over a DNS mergers, having the $\mathrm{BH}$ a rather large mass $\left(M_{\mathrm{bh}} \gtrsim 20 M_{\odot}\right)$ and being threaded by huge magnetic fields $\left(B \gtrsim 10^{16} \mathrm{G}\right)$. An electromagnetic energy extraction mechanism directly linked to the $\mathrm{BH}$ spin, like the BlandfordZnajek process, may likely operate in this subclass of GRBs. We note that only a factor of three smaller value of $B$, would request $M_{\mathrm{bh}} \gtrsim 60 M_{\odot}$ to explain the observed $E_{\text {iso. However, to form }}$ so massive BHs the progenitor star shall have a mass $\sim 140 M_{\odot}$, i.e., they are in the limit of being stars whose final fate is to be disrupted by a single pulse due to the pair instability, without leaving any remnant BH (Woosley et al. 2002), i.e., without the possibility of producing a GRB engine. Considering the fact that magnetic fields in excess of $\sim 10^{16} \mathrm{G}$ are difficult to build up by the collapse of the core of the progenitor star, and that such fields may reduce the angular momentum of the resulting $\mathrm{BH}$, our preferred choice of parameters is a combination of magnetic field strenght $B \gtrsim$ few $\times 10^{15} \mathrm{G}$ and $M_{\text {bh }} \gtrsim 50 M_{\odot}$. In regard of these figures, we temptatively suggest that systems with large $\mathrm{BH}$ masses (close to the limit set by the pair instability) and magnetar-magnetic field strengths may constitute the specific type I scenario invoking the BZ-mechanism in a $\mathrm{NS}+\mathrm{BH}$ mergers ocurring at high- $z$ considered by Zhang et al. (2009). However, we cannot rule out the possibility that a single massive low-metallicity star (type II scenario), rather than a NS+BH merger, that yields a $\mathrm{BH}$ with the aforementioned properties, and which fuels an ultrarelativistic ejecta by means of a BZmechanism, constitutes the central engine of GRB 080913.

It has been also demonstrated that the synergy between missions like Swift, automated instruments and robotic observatories, facilitate further study of the population of high-z GRBs, and help to assess whether a significant fraction of short-duration GRBs coexist at these high redshifts.

Acknowledgements. We thank the generous allocation of observing time by different Time Allocation Committees. This work is partially based on observations collected at the Centro Astronómico Hispano Alemán (CAHA) at Calar Alto, operated jointly by the Max-Planck Institut für Astronomie and the Instituto de Astrofísica de Andalucía (CSIC). IRAM is an international institute funded by the Centre National de la Recherche Scientifique (France), the Max Planck Gesellschaft (Germany) and the Instituto Geográfico Nacional (Spain). This work made use of data supplied by the UK Swift Science Data Centre at the University of Leicester and of data products from the Two Micron All Sky Survey (2MASS), 2MASS, which is a joint project of the Univ. of Massachusetts and the IR Processing and Analysis Center/CalTech, funded by NASA and NSF. We thank the assistance of D. Cristóbal Hornillos. DPR acknowledges support from the "José Castillejo" program. AdUP acknowledges support from an ESO fellowship. M.A.A. is a Ramón y Cajal Fellow. J.O. and K.P. acknowledge the support of the STFC. I.H. and V.P. acknowledge support from OTKA grants T48870 and K77795. This research has also been partially supported by the Spanish MICINN under the programmes AYA2007-63677, AYA200803467/ESP, AYA2007-67626-C03-01 and CSD2007-00050.

\section{References}

Akiyama, S., Wheeler, J. C., Meier, D. L., \& Lichtenstadt, I. 2003, ApJ, 584, 954 Aloy, M. A., Janka, H.-T., Müller, E. 2005, A\&A, 436, 273

Arimoto, M., Ricker, G., Atteia, J.-L., Kawai, \& N. 2006, GCN Circ., 4550

Bailer-Jones, C. A. L., Bizenberger, P., \& Storz, C. 2000, Proc. SPIE, 4008, 1305 Beardmore, A. P., Evans, P. A., Goad, M. R., \& Osborne, J. P. 2008, GCN Circ., 8219

Belczynski, K., Perna, R., Bulik, T., et al. 2006, ApJ, 648, 1110. Belczynski, K., Holz, D. E., Fryer, C. L., et al. 2010, ApJ, 708, 117 Berger, E. 2007, ApJ, 670, 1254

Berger, E., Price, P. A., Cenko, S. B., et al. 2005, Nature, 438, 988 Berger, E., Shin, M.-S., Mulchaey, J. S., \& Jeltema, T. E. 2007a, ApJ, 660, 496 Berger, E., Fox, D. B., Price, P. A., et al. 2007b, ApJ, 664, 1000 Birkl, R., Aloy, M. A., Janka, H.-Th., \& Müller, E. 2007, A\&A, 463, 51 Blandford, R. D., \& Znajek, R. L. 1977, MNRAS, 179, 433

Bloom, J. S., Perley, D. A., Chen, H.-W., et al. 2007, ApJ, 654, 878 D’Avanzo, P., Covino, S., Antonelli, L. A. et al. 2008, GCN Circ., 8220 Evans, P., Beardmore, A. P., Page, K. L., et al. 2007, A\&A, 469, 379 Fox, D. B., Frail, D. A., Price, P. A., et al. 2005, Nature, 437, 845 Fynbo, J., Greiner, J., Krueler, T., et al. 2008, GCN Circ., 8225 Gehrels, N., Sarazin, C. L., O’Brien, P. T., et al. 2005, Nature, 437, 851 Greiner, J., Kruehler, T., \& Rossi, A. 2008, GCN Circ., 8223 Greiner, J., Krühler, T., Fynbo, J. P. U., et al. 2009, ApJ, 693, 1610 Haislip, J. B., Nysenwander, M. C., Reichart, D. E., et al. 2006, Nature, 440, 181 Horváth, I., Balázs, L. G., Bagoly, Z., et al. 2006, A\&A 447, 23 Horváth, I., Balázs, L. G., Bagoly, Z., et al. 2008, A\&A 489, L1 Janka, H. -Th., Aloy, M. -A., Mazzali, P. A., \& Pian, E. 2006, ApJ, 645, 1305 Jóhannesson, G., Björnsson, G., \& Gudmundsson, E. H. 2006, ApJ, 647, 1238 Kalberla, P. M. W., Burton, W. B., Hartmann, D. et al. 2005, A\&A 440, 775 Kawai, N., Kosugi, G., Aoki, K., et al. 2006, Nature 440, 184 King, A., Olsson, E., \& Davies, M. B. 2007, MNRAS, 374, 34 Kouveliotou, C., Meegan, C. A., Fishman, G. J., et al. 1993, ApJ, 413, L101 Lee, H. K., Wijers, R. A. M. J., \& Brown, G. E. 2000, Phys. Rep., 325, 83 Lee, W.-H., Ramirez-Ruiz, E., \& Granot, J. 2005, ApJ, 630, L165 Mimica, M., \& Aloy, M. A. 2010, MNRAS, 401, 525 Obergaulinger, M., Aloy, M. A., \& Müller, E. 2006a, A\&A, 450, 1107

Obergaulinger, M., Aloy, M. A., Dimmelmeier, H., \& Müller, E. 2006b, A\&A, 457,209

Obergaulinger, M., Cerdá-Durán, P., Müller, E., \& Aloy, M. A. 2009, A\&A, 498, 214

Oechslin, R., \& Janka, H.-T. 2006, MNRAS, 368, 1489.

Oechslin, R., Janka, H.-T., \& Marek, A. 2007, A\&A, 467, 395

Pal'shin, V., Golenetskii, S., Aptekar, R., et al. 2008, GCN Circ., 8256

Panaitescu, A., \& Kumar, P. 2001, ApJ, 554, 667

Popham, R., Woosley, S. E., \& Fryer, C. 1999, ApJ, 518, 356

Rossi, A., Greiner, J., Kruehler, T., et al. 2008, GCN Circ., 8218

Ruffert M., \& Janka, H.-T. 1999, A\&A, 344, 573

Salvaterra, N., Della Valle, M., Campana, S., et al. 2009, Nature, 461, 1258

Sari, R., Piran, T., \& Halpern, J. P. 1999, ApJ, 519, L17

Setiawan, S., Ruffert, M., \& Janka, H.-Th. 2006, A\&A, 458, 553

Schady, P., Beardmore, A. P., Cummings, J. R., et al. 2008, GCN Circ., 8217

Shibata, M., \& Taniguchi, K. 2008, PhRvD, 77(8), ID 080415

Soderberg, A. M., Berger, E., Kasliwal, M., et al. 2006, ApJ, 650, 261

Stamatikos, M., et al. 2008, GCN Circ., 8222

Tanvir, N., Fox, D. B., Levan, A. J., et al. 2009, Nature, 461, 1254 de Ugarte Postigo, A., Castro-Tirado, A. J., Guziy, S., et al. 2006, ApJ, 648, L83

Xu, D. 2008, GCN Circ., 8267

Woosley, S. E., Heger, A., \& Weaver, T. A. 2002, Rev. Mod. Phys., 74, 1015

Yoshida, N., Kazuyuki, O., \& Hernquist, L. 2008, Science, 321, 669

Zhang, B., Zhang, B.-B., Virgili, F. J. et al. 2009, ApJ, 703, 1696 